\title{
Connaissances et perception des entraîneurs au sujet de la consommation de tabac à chiquer chez les jeunes athlètes : résultats d'un sondage réalisé en 2009 en Ontario
}

\author{
J. H. C. Skinner, M. Sc. (1); S. J. Bobbili, M.H.P. (2)
}

Cet article a fait l'objet d'une évaluation par les pairs.

\section{Résumé}

Introduction : Les professionnels de la santé publique se préoccupent de la progression de la consommation de tabac à chiquer (TC) par les athlètes. On dispose toutefois de peu de données sur la situation au Canada, notamment chez les jeunes.

Méthodologie : La coalition Pas aux jeunes et la Coaches Association of Ontario ont effectué un sondage auprès des entraîneurs afin d'évaluer, d'une part, le degré de connaissance et de sensibilisation de ces derniers au sujet du TC et, d'autre part, la perception qu'ont ces derniers du rôle que peut jouer l'entraîneur pour influencer la consommation de TC chez les athlètes qu'ils encadrent. Les sondages ont été acheminés par voie électronique à des entraîneurs encadrant des garçons et des filles âgés de 9 à 18 ans pratiquant le baseball, le basketball, le football, le soccer et l'athlétisme en Ontario.

Résultats : Presque tous les entraîneurs interrogés ont répondu correctement aux questions sur les effets de la consommation de TC sur la santé et environ $80 \%$ d'entre eux ont répondu correctement à la question portant sur les dispositions législatives relatives au TC et aux jeunes.

Conclusion : La plupart des entraîneurs souhaiteraient recevoir de l'information sur le TC, en particulier sur les effets de la consommation de TC sur la santé et sur les façons de prévenir la consommation de TC chez les athlètes. L'information fournie aux entraîneurs devrait revêtir plusieurs formes et comprendre des documents électroniques et imprimés.

Mots-clés : tabac, tabac sans fumée, tabac à chiquer, tabac à mâcher, jeunes, sport, mentor, entraîneur

\section{Introduction}

D'après certaines observations et les résultats de sondages locaux communiqués lors de réunions de la coalition Pas aux jeunes (PAJ) en 2006 et en 2007, la consommation de tabac « oral » (tabac à chiquer [TC]) serait en hausse chez les participants à des activités sportives ${ }^{1}$. Notre recherche vise à déterminer le niveau de connaissance des entraîneurs de l'Ontario au sujet du tabac à chiquer et à évaluer la perception qu'ont ces entraîneurs de la consommation de TC par les athlètes qu'ils encadrent. La présente étude n'a pas pour objectif de mesurer la prévalence de la consommation, mais plutôt d'orienter les initiatives de promotion de la santé visant à prévenir le début de la consommation de TC par les jeunes et à promouvoir l'abandon de cette consommation. L'étude a été réalisée au moyen d'un sondage en ligne auprès d'entraîneurs amateurs encadrant des enfants et des adolescents en Ontario.

Notre projet s'inscrit directement dans le cadre des dispositions législatives adoptées par le gouvernement de l'Ontario, lesquelles demandent de travailler avec les populations prioritaires afin qu'elles adoptent un mode de vie sans tabac et afin de réduire le fardeau des maladies chroniques évitables. Il répond également à l'exigence des conseils de santé de surveiller les nouvelles tendances dans la consommation de tabac ${ }^{2}$.

\section{Contexte}

Tabac à chiquer : consommation et risques

Le tabac sans fumée se définit comme un produit du tabac consommé sans combustion $^{3}$. Le tabac sans fumée se présente principalement sous deux formes : le tabac à sucer (tabac à priser en poudre et humidifié, pour la bouche) et le tabac à chiquer (haché grossièrement) ${ }^{4}$. Les personnes qui consomment du tabac sans fumée soit placent une " pincée " de tabac à sucer entre la gencive et la lèvre ou la joue, soit mâchent une " chique » de tabac à chiquer. La nicotine ainsi libérée est absorbée par les muqueuses de la bouche ${ }^{4}$. Le terme " tabac à chiquer » (TC) utilisé dans cet article renvoie à ces deux types de tabac.

À l'instar de tous les produits du tabac, le TC est associé à de nombreux effets néfastes pour la santé. Les nitrosamines spécifiques

Rattachement des auteurs :

1. Circonscription sanitaire du district de Haliburton, Kawartha et Pine Ridge, Port Hope (Ontario), Canada

2. Centre de toxicomanie et de santé mentale, Toronto (Ontario), Canada

Correspondance : Jennifer Hope Campin Skinner, Circonscription sanitaire du district de Haliburton, Kawartha et Pine Ridge, 200 Rose Glen Road, Port Hope (Ontario) L1A 3V6; tél. : 905-885-9100; téléc. : 905-885-9551; courriel : jskinner@hkpr.on.ca 
du tabac (substances chimiques cancérogènes) ont été associées aux cancers de la bouche chez l'humain ${ }^{5}$. Il a notamment été établi que la consommation de TC est à l'origine de la leucoplasie, soit la présence de plaques et de lésions blanches à l'intérieur des joues, sur les gencives ou sur la langue pouvant causer le cancer de la bouche ${ }^{5}$. La consommation de TC peut également provoquer des maladies des gencives et la carie dentaire et elle est associée à un plus grand nombre de décès par maladie cardiovasculaire et par accident vasculaire cérébral $(\mathrm{AVC})^{6,7}$. Fait particulièrement préoccupant, le TC peut engendrer une dépendance plus forte que la cigarette. Par rapport aux fumeurs de cigarettes, les consommateurs de TC sont exposés à de grandes quantités de nicotine pendant de plus longues périodes $^{5}$.

\section{Consommation de tabac à chiquer par les jeunes}

La coalition PAJ est un réseau de 31 bureaux de santé publique en Ontario qui a pour objectif de réduire l'accès des jeunes aux produits du tabac et à leur consommation par les jeunes. PAJ a récemment mené des sondages et créé des groupes de discussion ciblant les Ontariens âgés de 14 à 19 ans. Il en est ressorti que ces jeunes percevaient le TC comme un produit du tabac plus sécuritaire que la cigarette du fait qu'il ne produisait aucune fumée ${ }^{1}$. De plus, les répondants n'ont pas réussi à reconnaître de nombreux effets de la consommation de TC sur la santé1.

En Amérique du Nord et en Europe, la consommation de TC n'est pas aussi répandue que celle de la cigarette, mais elle pourrait augmenter en raison de la mise en place des interdictions de fumer dans les lieux publics ${ }^{7}$. Selon l'Enquête de surveillance de l'usage du tabac au Canada de 2008, $1 \%$ des jeunes de 15 à 19 ans $(n=30000)$ et $1 \%$ des jeunes adultes de 20 à 24 ans $(\mathrm{n}=27$ 000) ont déclaré avoir consommé du TC au cours des 30 derniers jours $^{8}$. Toutefois, dans l'Enquête sur le tabagisme chez les jeunes de 2008-2009, $5 \%$ des élèves canadiens de la $\sigma^{\text {e }}$ à la $12^{\mathrm{e}}$ année ( $\left.\mathrm{n}=2600\right)$ et $4 \%$ des élèves ontariens de la $6^{\mathrm{e}}$ à la $12^{\mathrm{e}}$ année $(\mathrm{n}=360)$ ont déclaré avoir « déjà essayé » le $\mathrm{TC}^{9}$.
Aux États-Unis, la National Survey on Drug Use and Health a révélé que la consommation de TC chez les personnes âgées de 12 ans et plus était demeurée stable, entre $3,0 \%$ et 3,3\%, entre 2002 et $2007^{10}$. La consommation de TC avait néanmoins augmenté parmi certaines sous-populations, notamment les garçons adolescents, chez qui elle était passée de $3,4 \%$ en 2002 à 4,4 \% en 2007, et elle était plus élevée parmi ceux vivant en milieu rural ${ }^{10}$.

Lâge, le sexe, le lieu de résidence, l'origine ethnique et la scolarité sont tous des facteurs liés à la prévalence de la consommation de TC. Selon les données de la National Youth Risk Behavior Survey de 2009, la prévalence de la consommation de TC chez les élèves américains du secondaire était estimée à 8,9 \% ${ }^{11}$. Cette prévalence variait selon les États, allant de 4,9 \% à Hawaï à 16,2\% au Wyoming; elle était plus élevée chez les garçons que chez les filles du secondaire, et chez les élèves blancs que chez les élèves noirs et hispaniques $^{11}$. La prévalence chez les garçons blancs était très élevée : 20,1 \% ${ }^{11}$.

Goebel et collab. ont dégagé plusieurs corrélats de la consommation de TC chez les élèves américains de $5^{\mathrm{e}}$, de $8^{\mathrm{e}}$ et de $11^{\mathrm{e}}$ année, dont le fait d'avoir un membre de la famille n'habitant pas à la résidence familiale qui consomme du TC, d'avoir un ami qui consomme du TC, de jouer au football, d'avoir déjà essayé la cigarette et d'avoir des parents qui autorisent la consommation de TC à la maison $^{12}$.

\section{La consommation de tabac à chiquer chez les sportifs}

Pour connaître les variations de consommation de TC selon les sports, la National Collegiate Athletic Association (NCAA) a demandé en 2001 à des athlètes de sexe masculin (de 17 à 20 ans) d'indiquer leur consommation de TC au cours des 30 derniers jours. Les résultats furent les suivants : baseball, $41 \%$; lutte, $39 \%$; hockey sur glace, $35 \%$; crosse, $32 \%$; football, $29 \%$; golf, $27 \%$; water-polo, $25 \%$; soccer, $20 \%$; athlétisme, $17 \%$; tennis, $13 \%$; basketball, $12 \%{ }^{13}$.
Plusieurs études indiquent que les adolescents qui participent à des sports organisés sont moins nombreux à fumer la cigarette, mais plus nombreux à consommer du $\mathrm{TC}^{14,15}$. Castrucci et collab. ont révélé que les adolescents de $9^{e}$ à $12^{\mathrm{e}}$ année qui participent à des sports organisés courent un risque $33 \%$ plus élevé d'avoir déjà consommé du TC et un risque $76 \%$ plus élevé de consommer du $\mathrm{TC}^{14}$. Rigotti et collab. ont présenté des résultats semblables dans une étude auprès de 14138 étudiants de 18 à 24 ans fréquentant 119 collèges aux États-Unis ${ }^{16}$. Les chercheurs ont observé que les athlètes pratiquant un sport intercollégial étaient plus susceptibles de consommer du TC que de fumer des cigarettes; ils ont avancé que les athlètes pourraient consommer du TC comme substitut nicotinique à la cigarette $^{16}$.

La NCAA a réalisé une étude auprès de 1985 équipes en faisant appel à 1032 établissements membres et a obtenu 19676 réponses, ce qui lui a permis d'établir la prévalence de la consommation de TC à 16,3 \% ${ }^{17}$. Parmi les athlètes qui consomment du TC, plus de $50 \%$ ont commencé au secondaire, cependant environ $10 \%$ ont commencé au moment où ils fréquentaient l'école intermédiaire ou plus tôt ${ }^{17}$.

Les étudiants-athlètes de niveau collégial ont indiqué consommer des drogues à usage récréatif, comme le $\mathrm{TC}$, principalement pour des raisons sociales et récréatives $(46,8 \%)$ ou pour se sentir bien $(28,1 \%)^{18}$. Les autres raisons invoquées était de mieux composer avec le stress de la vie d'étudiant et d'athlète au collège (21,2 \%), d'améliorer leurs performances sportives $(2,0 \%)$ et de s'intégrer à l'équipe $(1,8 \%)^{17}$.

\section{Le rôle des entraîneurs}

Les entraîneurs jouent un rôle d'enseignant, de mentor, de modèle, d'ami et de leader dans la communauté ${ }^{19}$. Ils peuvent grandement orienter les valeurs et les normes de l'équipe et, en tant que modèles, peuvent exercer une influence déterminante sur le façonnement des habitudes des enfants et des jeunes adultes ${ }^{20}$. 
Walsh et collab. ont confirmé l'influence que les entraîneurs exercent sur les athlètes, en particulier ce qui concerne la consommation de $\mathrm{TC}^{21}$. Leurs résultats révèlent que les jeunes garçons du secondaire qui jouent au baseball sont trois à quatre fois plus à risque de consommer du TC s'ils ont vu l'entraîneur de leur école secondaire ou leur père consommer ces produits ${ }^{21}$.

Les entraîneurs peuvent tout autant inciter les jeunes à commencer à consommer du TC que les dissuader d'en consommer. Ils jouent ainsi un rôle clé dans les efforts de réduction de la consommation de TC chez les athlètes, car ils côtoient des joueurs à différents stades de consommation de TC (p. ex. début, expérimentation, consommation régulière) ${ }^{22}$. Parmi les entraîneurs interrogés par Horn et collab., $80 \%$ ont reconnu jouer un rôle déterminant dans la prévention de la consommation de TC chez les athlètes, tandis que $86 \%$ se croyaient en mesure d'aider les jeunes à mettre un terme à leur consommation ${ }^{22}$. Les entraîneurs ont mentionné recourir à plusieurs stratégies en vue de contrer la consommation de TC chez leurs athlètes, notamment en conseillant à ces derniers de cesser leur consommation, en les informant des dangers du TC pour la santé et en mettant les parents ou la direction de l'école au courant de la situation ${ }^{22}$.

\section{Méthodologie}

En partenariat avec la Coaches Association of Ontario (CAO), un organisme sans but lucratif dirigé par des entraîneurs et offrant des possibilités de perfectionnement et des ressources éducatives aux entrâ̂neurs, PAJ a conçu un sondage qui a été acheminé par voie électronique à des entraîneurs amateurs en juin 2009. Le but du sondage était 1) d'évaluer la connaissance générale et la sensibilisation des entraîneurs au sujet de la consommation de TC et 2) de mieux comprendre la perception qu'ont les entraîneurs du rôle qu'ils peuvent jouer pour influencer leurs athlètes et prévenir la consommation de TC chez ces derniers.

Tous les entraîneurs interrogés étaient membres d'organismes de sport amateur. On présume donc qu'ils étaient bénévoles (non rémunérés) à tous les niveaux et pour tous les sports et qu'ils encadraient leurs athlètes pendant leur temps libre.

Des membres de la CAO et de PAJ, de concert avec un épidémiologiste, ont choisi les critères de participation au sondage et ont élaboré le sondage par un processus itératif de définition de contenu et de reformulation des questions. Les critères d'admissibilité ainsi que les raisons justifiant l'inclusion ou l'exclusion des entraîneurs étaient les suivants :

- Les participants étaient âgés d'au moins 18 ans. Pour les personnes de moins de 18 ans, la procédure de consentement aurait été plus complexe.

- Les participants étaient entraîneurs dans au moins une des disciplines suivantes: hockey, baseball, soccer, basketball, football ou athlétisme. La CAO, véhicule principal de la collecte de données initiale, ne comptait parmi ses membres que des entraîneurs de ces disciplines.

- Les participants encadraient des jeunes de 9 à 18 ans. Cet intervalle d’âge étendu a été choisi car il existe peu d'études sur la consommation de TC chez les jeunes athlètes et qu'aucune n'a jamais englobé de jeunes de moins de 12 ans.

Le questionnaire a été envoyé par voie électronique au moyen de SurveyMonkey, un outil d'élaboration de sondages par Internet. Les réponses étaient encodées et hébergées sur le serveur Web de SurveyMonkey.

Les membres de la CAO qui répondaient aux critères d'admissibilité constituaient la population cible initiale. La taille de l'échantillon a été estimée à 321 personnes, compte tenu du nombre total de membres de la CAO, d'une marge d'erreur de $5 \%$ et d'un intervalle de confiance à 95 \% (tableau 1).

La CAO a informé ses membres du projet et a fourni le lien vers le sondage dans son bulletin électronique mensuel du 11 juin 2009. En participant, les répondants couraient la chance gagner l'un des quatre bons-cadeaux de 50 \$ ou divers articles de promotion antitabac, les gagnants étant choisis par tirage au sort après la date de fin du sondage.

Deux semaines après l'envoi du bulletin électronique initial par la CAO, 79 personnes avaient répondu au sondage. Un rappel au sujet du sondage a été ajouté au bulletin électronique de juillet destiné aux membres de la CAO. À la suite de cette communication, le taux de réponse est demeuré faible. Par conséquent, le lien vers le sondage a également été envoyé aux organismes provinciaux régissant les sports ciblés : Ontario Baseball Association, Ontario Basketball Association, Ontario Football Alliance, Ontario Hockey Federation, Ontario Soccer Association et Athletics Ontario. La CAO a officiellement demandé à ces organismes de fournir le lien à leurs membres dans leur propre bulletin électronique. Un mois après la communication initiale, 270 questionnaires avaient été remplis. Pour augmenter le nombre de réponses, le lien menant au sondage a alors été fourni aux membres de PAJ pour qu'ils le diffusent auprès de leurs organismes locaux de sport amateur. En élargissant ainsi la population, la signification statistique ne pouvait plus être

TABLEAU 1

Membres de la CAO participant à un sondage sur la consommation de tabac à chiquer chez les jeunes athlètes

\begin{tabular}{|c|c|c|}
\hline \multirow[t]{2}{*}{ Sport } & \multicolumn{2}{|c|}{ Entraîneurs (n) } \\
\hline & Membres de la CAO & $\begin{array}{l}\text { Taille de l'échantillon requise } \\
\text { pour l'analyse des sous-groupes }\end{array}$ \\
\hline Baseball & 124 & 94 \\
\hline Basketball & 969 & 276 \\
\hline Football & 119 & 92 \\
\hline Hockey & 244 & 150 \\
\hline Soccer & 353 & 185 \\
\hline Athlétisme & 113 & 88 \\
\hline Total & 1922 & 321 \\
\hline
\end{tabular}

Abréviation : CAO, Coaches Association of Ontario.

${ }^{a}$ Estimation de la taille de l'échantillon fondée sur une marge d'erreur de $5 \%$ et un intervalle de confiance à $95 \%$. 
calculée. Toutefois, cette dernière n'aurait pu être atteinte à partir du taux de réponse obtenu des seuls membres de la CAO en raison de la petite taille de l'échantillon. On reconnaît également que le fait de modifier la stratégie de collecte de données peut induire un biais géographique inhérent dans la population (p. ex., certaines régions sanitaires peuvent faire plus de promotion que d'autres), mais il fallait néanmoins augmenter le nombre de répondants.

Une analyse descriptive comprenant les fréquences et des tableaux croisés a été réalisée à l'aide de SurveyMonkey et de Microsoft Excel 2000.

\section{Résultats}

Sur le serveur Web de SurveyMonkey, 344 questionnaires ont été remplis. De ce nombre, 83 ont été exclus parce que les répondants ne respectaient pas les critères d'admissibilité (entraîneur dans un sport autre que ceux ciblés, $n=70$; entraîneur ayant indiqué n'encadrer que des athlètes de 8 ans ou moins, $\mathrm{n}=9$, ou de 19 ans et plus, $n=4)$. Les réponses aux 261 questionnaires restants ont été analysées au moyen de statistiques descriptives.

\section{Entraîneurs}

Les répondants étaient majoritairement âgés de 35 à 54 ans (72\%), environ $10 \%$ étaient âgés de 55 ans et plus et les $20 \%$ restant étaient âgés de 18 à 24 ans. Il s'agissait surtout d'hommes (76\%).

\section{Origine géographique}

Le tableau 2 présente la répartition géographique des entraîneurs. Les zones géographiques sont fondées sur les réseaux régionaux antitabac (RRA) que le ministère de la Promotion de la santé et du Sport a créés, dans le cadre de la stratégie Ontario sans fumée, afin de coordonner les initiatives régionales, de favoriser l'utilisation de ressources limitées et d'adapter les activités à des contextes particuliers ${ }^{23}$.

\section{Sport}

La répartition des entraîneurs selon le sport était inégale. Plus du quart (29 \%) des répondants ont déclaré être principalement
TABLEAU 2

Répartition géographique des entraîneurs interrogés, Ontario (Canada)

\begin{tabular}{lc} 
Région (RRA) & Pourcentage (\%) \\
\hline Centre-Est & 29 \\
Centre-Ouest & 34 \\
Est & 9 \\
Nord-Est & 5 \\
Nord-Ouest & 1 \\
Sud-Ouest & 13 \\
Toronto & 9 \\
Inconnu & 2 \\
\hline
\end{tabular}

Abréviation : RRA, réseau régional antitabac.

entraîneurs de baseball et environ le même pourcentage (28 \%), être entraîneurs de soccer. Les entraîneurs de basketball (17 \%) et de hockey (13\%) étaient également bien représentés. Les entraîneurs de football représentaient moins de $10 \%$ de l'échantillon, tout comme ceux d'athlétisme.

\section{Niveau de compétition}

La plupart des entraîneurs ont déclaré encadrer des athlètes à plus d'un niveau de compétition, majoritairement à un niveau de compétition (provincial) (59\%) et pour des sports récréatifs (43\%).

\section{Fréquence et durée de la fonction d'entraîneur}

Les entraîneurs de tous les niveaux de compétition ont déclaré exercer leurs fonctions deux mois ou plus par année. Le tiers (32 \%) des entraîneurs ont indiqué encadrer leur équipe depuis trois à cinq ans, alors que $18 \%$ des répondants étaient entraîneurs depuis moins d'un an, et $26 \%$ depuis six ans ou plus.

\section{Athlètes}

La plupart des entraîneurs ont déclaré encadrer des athlètes de nombreuses catégories d'âge, principalement des jeunes de 13 à 14 ans. Un peu plus de la moitié des entraîneurs (51 \%) ont indiqué encadrer des équipes masculines, mais l'échantillon comprenait aussi des entraîneurs d'équipes féminines (31\%) et d'équipes mixtes (26\%).

\section{Connaissance subjective du TC}

La plupart des entraîneurs (68%) ont déclaré avoir au moins une connaissance minimale du TC. Les entraîneurs de 45 ans et plus étaient plus nombreux que leurs homologues âgés de moins de 45 ans à avoir au moins une connaissance minimale du TC, soit $74 \%$ contre $58 \%$.

\section{Moyens de se procurer du TC}

De nombreux entraîneurs (29 \%) pensent que les athlètes achètent les produits du TC directement des vendeurs, p. ex. les dépanneurs, tandis qu'un pourcentage équivalent pensent que ce sont des amis, des membres de la famille ou des connaissances qui donnent ces produits aux athlètes. Certains entraîneurs (15 \%) pensent que les athlètes obtiennent ces produits sur le " marché gris » (p. ex. sur Internet, dans la rue), $6 \%$ pensent que les athlètes les subtilisent à la maison, $3 \%$ ont choisi la réponse " Autre » et $18 \%$ pensent que les athlètes ne se procurent pas de tels produits.

\section{Perception de la prévalence de la consommation de TC chez les athlètes}

La plupart des entraîneurs (78 \%) ont indiqué qu'aucun joueur de leur équipe ne consommait de TC. Environ $9 \%$ ont déclaré soit avoir vu leurs joueurs en consommer, soit soupçonner qu'un ou plusieurs joueurs en consommaient, tandis que $5 \%$ ont indiqué avoir vu des joueurs d'autres équipes en consommer. Dix pour cent des répondants ont déclaré ne pas savoir si leurs joueurs consommaient du TC.

Seuls les entraîneurs encadrant des jeunes de 13 ans et plus ont déclaré savoir que des jeunes de leur équipe consommaient du TC. Cependant, quelques entraîneurs 
encadrant des jeunes de 9 à 12 ans ont indiqué soupçonner que certains d'entre eux consommaient du TC ou avoir été témoin de la consommation de TC par des athlètes d'autres équipes du même groupe d'âge.

Tous les entraîneurs ayant déclaré avoir $v u$ un ou plusieurs de leurs joueurs consommer du TC encadraient des équipes masculines. Seuls des entraîneurs de baseball, de football et de hockey ont indiqué avoir été témoin d'une consommation de TC par des athlètes de leur équipe.

\section{Connaissances sur le TC et la consommation de TC}

La section "Vrai ou faux » du sondage contenait des questions sur les connaissances des entraîneurs au sujet des produits du TC et de la consommation de ces produits. La majorité des entraîneurs a indiqué avec justesse que le TC n'améliorait pas les performances sportives (98 \%), qu'il ne constituait pas une solution de rechange sécuritaire à la cigarette (98\%) et qu'il pouvait causer des lésions buccales, une récession gingivale et la perte des dents (98\%). Parmi les répondants, $96 \%$ ont indiqué avec justesse que la consommation de TC pouvait contribuer à l'hypertension et à la survenue de crises cardiaques et d'AVC, $52 \%$ étaient d'avis que le tabac à chiquer constituait, à l'heure actuelle, un problème grandissant parmi les jeunes faisant du sport en Ontario, et $78 \%$ ont déclaré avec justesse qu'il est illégal de donner du TC à des jeunes de moins de 19 ans.

\section{Rôle perçu dans la lutte contre la consommation de TC}

Les entraîneurs devaient indiquer quel était, à leur avis, leur rôle dans la lutte contre le TC au sein de leur équipe. Plus de la moitié d'entre eux ont déclaré qu'ils rencontreraient les athlètes et les conseilleraient (56 \%) ou qu'ils aviseraient les parents des athlètes (54 \%). Par ailleurs, de nombreux entraîneurs ont indiqué qu'ils donneraient aux athlètes de la documentation sur le TC (38 \%). Certains (36 \%) ont déclaré qu'ils aiguilleraient les athlètes vers des services de soutien (p. ex. fournisseurs de soins de santé, professionnels de la santé publique ou représentants sportifs) ou informeraient l'organisateur de la ligue ou des organismes communautaires (p. ex. ligue, école) de toute consommation de TC (25\%).

\section{Stratégies utilisées pour lutter contre la consommation de TC}

Près de la moitié des répondants (45\%) ont déclaré n'avoir rien fait pour influencer leurs athlètes au sujet de la consommation de TC et ne pas s'être préoccupés de cette question parce que le problème ne se posait pas. Plusieurs entraîneurs, en particulier ceux encadrant des équipes féminines, ont indiqué n'avoir pas vu la nécessité de s'attaquer à cette question puisque leurs athlètes étaient trop jeunes. Environ le quart (23 \%) des répondants ont déclaré promouvoir ou appliquer une politique de sport sans tabac.

\section{Information sur le TC jugée utile par les entraîneurs}

On a demandé aux entraîneurs d'indiquer le type d'information qui leur serait le plus utile pour conseiller les jeunes au sujet de la consommation de TC. Près de la moitié (47\%) des répondants ont déclaré que l'information sur les effets du TC sur la santé serait le plus utile (tableau 3).

\section{Préférences quant au mode de transmission de l'information sur le TC}

De nombreux entraîneurs ont indiqué qu'ils préféreraient voir l'information sur le TC figurer dans le site Web de la CAO (40\%), dans leur manuel des entraîneurs ou dans des bulletins d'information et des brochures (tableau 4). Comme un grand nombre de répondants étaient membres de la CAO et avaient vraisemblablement eu accès au sondage par l'intermédiaire du site Web de la CAO, la préférence pour ce mode de transmission pourrait ne pas s'appliquer à l'ensemble de la population des entraîneurs. Des répondants, peu nombreux, ont déclaré qu'ils préféreraient assister à des ateliers pour en apprendre davantage sur cette question. Dans la catégorie "Autre », la plupart des répondants ont indiqué préférer que l'information leur soit transmise par courriel (par leur club sportif ou la CAO).

\section{Analyse}

Notre étude visait, d'une part, à déterminer ce que les entraîneurs savaient à propos de la consommation de TC chez les athlètes qu'ils encadrent et, d'autre part, à dégager leurs lacunes sur le plan des connaissances en matière de consommation de TC.

La majorité des répondants a déclaré n'avoir vu aucun athlète consommer de TC. Puisque notre étude visait à orienter les activités de promotion de la santé plutôt

TABLEAU 3

Type d'information sur le tabac à chiquer jugée utile par les entraîneurs, selon la région (RRA), Ontario (Canada)

\begin{tabular}{|c|c|c|c|c|c|c|c|c|}
\hline \multirow[t]{2}{*}{ Type d'information jugée utile } & \multicolumn{8}{|c|}{ Entraîneurs, selon l'origine géographique par RRA (\%) } \\
\hline & Centre-Est & Centre-Ouest & Est & Nord-Est & Nord-Ouest & Sud-Ouest & Toronto & Ensemble \\
\hline Les différentes formes de TC & 29 & 22 & 25 & 13 & 0 & 29 & 34 & 26 \\
\hline $\begin{array}{l}\text { Les moyens d'inciter les athlètes } \\
\text { à éviter le TC }\end{array}$ & 36 & 35 & 25 & 47 & 0 & 38 & 48 & 36 \\
\hline $\begin{array}{l}\text { Les manières dont l'industrie } \\
\text { du tabac cible les jeunes }\end{array}$ & 28 & 21 & 43 & 20 & 0 & 31 & 31 & 27 \\
\hline $\begin{array}{l}\text { Je n'ai pas besoin d'information } \\
\text { en ce moment }\end{array}$ & 24 & 31 & 21 & 27 & 33 & 26 & 7 & 25 \\
\hline
\end{tabular}

Abréviations : RRA, réseau régional antitabac; TC, tabac à chiquer. 
TABLEAU 4

Sources d'information préférées des entraîneurs au sujet du tabac à chiquer, selon la région (RRA), Ontario (Canada)

\begin{tabular}{|c|c|c|c|c|c|c|c|c|}
\hline \multirow[t]{2}{*}{ Source d'information préférée } & \multicolumn{8}{|c|}{ Entraîneurs, selon l'origine géographique par RRA, \% } \\
\hline & Centre-Est & Centre-Ouest & Est & Nord-Est & Nord-Ouest & Sud-Ouest & Toronto & Ensemble \\
\hline Site Web de la CAO & 45 & 35 & 54 & 47 & 0 & 29 & 55 & 40 \\
\hline Ateliers & 5 & 3 & 7 & 7 & 0 & 2 & 3 & 4 \\
\hline Bulletins et brochures & 31 & 37 & 46 & 7 & 0 & 38 & 34 & 34 \\
\hline
\end{tabular}

Abréviations : CAO, Coaches Association of Ontario; RRA, réseau régional antitabac.

qu'à fournir une estimation de la prévalence, les résultats que nous avons obtenus ne sont pas comparables à ceux d'études antérieures ayant porté sur la consommation autodéclarée de TC chez des athlètes ${ }^{13,17}$. D'autres facteurs associés à des différences entre les populations étudiées (p. ex. âge, origine géographique et culture) peuvent nuire encore davantage à la comparaison avec les études de prévalence antérieures sur le TC. Toutefois, notre constatation selon laquelle seuls les entraîneurs d'équipes masculines ont observé la consommation de TC chez des athlètes qu'ils encadrent concorde avec les résultats d'autres études.

De nombreux entraîneurs ont dit n'avoir rien fait pour s'attaquer à la question de la consommation de TC au sein de leur équipe. Cependant, loin d'être un signe de désintérêt envers les athlètes qu'ils encadrent, cette réalité traduit plutôt le fait que la plupart des entraîneurs ne voient pas la nécessité de lutter contre la consommation de TC parce qu'à leur avis ce problème ne se pose pas au sein de leur équipe. Il se peut que notre étude révèle que les entraîneurs ne sont pas vraiment au courant de la consommation de TC chez leurs athlètes.

Les entraîneurs âgés de 45 ans et plus affirment en savoir davantage sur les produits du TC et sur la consommation de TC que leurs homologues plus jeunes. En plus de l'âge, certaines caractéristiques des entraîneurs et des athlètes doivent être prises en compte puisqu'elles sont liées à des constatations relatives aux connaissances et aux perceptions des entraîneurs au sujet du TC. Par exemple, les résultats de la présente étude s'appliquent surtout aux équipes masculines de baseball, de basketball, de soccer et de hockey.

Bien que de nombreux entraîneurs aient déclaré ne pas avoir de problème de consommation de TC au sein de leurs équipes, près des trois quarts des entraîneurs ont affirmé qu'ils souhaiteraient obtenir plus d'information à ce sujet. Les résultats de l'étude offrent un aperçu des méthodes que les entraîneurs utilisent actuellement pour lutter contre la consommation de TC chez les joueurs qu'ils encadrent.

\section{Points forts et limites}

Au départ, le lien vers le sondage a été envoyé uniquement aux entraîneurs qui étaient des membres actifs de la CAO. Au fur et à mesure que la collecte de données avançait, il est devenu évident qu'on ne pourrait atteindre la cible fixée quant à la taille de l'échantillon; la stratégie d'échantillonnage a donc été modifiée. Le lien vers le sondage a alors été fourni à une population beaucoup plus vaste et la diffusion n'a pas été uniforme au sein de la province. Par conséquent, il se pourrait que les répondants soient concentrés en grappes, ce qui entraînerait, pour certaines réponses, une plus grande similarité que ce à quoi on s'attendrait avec un échantillon aléatoire. Étant donné qu'une vaste diffusion a eu lieu et qu'on ne peut savoir qui a reçu le lien vers le sondage, il se peut qu'on ne puisse généraliser les résultats à une population plus vaste d'entraîneurs.

Parmi les 36 bureaux de santé publique de l'Ontario, 31 sont membres de PAJ. De ce fait, et en raison de l'adoption, par les bureaux membres, de stratégies différentes en matière de promotion du sondage auprès des entraîneurs, la représentation des diverses régions de l'Ontario est inégale. Par conséquent, il se pourrait qu'on ne puisse généraliser les résultats de ce sondage, notamment aux régions géographiques sous-représentées.

Puisque la stratégie d'échantillonnage a été modifiée pendant la collecte de données, il n'est pas possible d'établir un taux de réponse. Toutefois, comme le taux de réponse des membres de la CAO a été extrêmement faible au départ, la diffusion électronique de sondages n'est peut-être pas la stratégie de collecte de données la plus efficace pour cette population. Les sondages en ligne comportent une autre limite, soit l'introduction possible d'un biais parmi les répondants. Par exemple, une grande proportion de répondants ont indiqué préférer recevoir l'information par l'intermédiaire du site Web de la CAO; toutefois, les personnes qui répondent à des sondages en ligne risquent davantage d'être plus familières avec l'environnement virtuel que la population d'entraîneurs dans son ensemble.

Les programmes des sondages Web ont comme limite inhérente un certain manque de sûreté concernant les données. Aucun renseignement signalétique ou personnel n'a été recueilli dans le cadre de cette étude et toutes les données ont été chiffrées.

Malgré les limites associées à la diffusion des sondages électroniques, cette méthode de collecte de données est efficace et peu coûteuse et se prête donc bien aux études comprenant un vaste échantillon de répondants géographiquement dispersés, comme dans le cas de la présente étude. Autre avantage, les sondages en ligne 
comprennent des dates de début et de fin bien définies, ce qui permet d'analyser les résultats dès la fin de la période de collecte de données.

Malgré ses limites, la présente étude est l'une des premières au Canada à faire la lumière sur cet enjeu à la fois récent et important.

\section{Conclusion}

D’après les résultats du sondage, les entraîneurs connaissent assez bien les effets des produits du TC et de la consommation de TC : presque tous les entraîneurs ont répondu correctement aux questions sur les effets de la consommation de TC sur la santé et environ $80 \%$ d'entre eux ont répondu correctement aux questions portant sur les dispositions législatives relatives au TC et aux jeunes.

La plupart des entraîneurs souhaitent recevoir de l'information sur le TC, notamment sur les effets de la consommation de TC sur la santé et sur la façon de prévenir la consommation de TC chez les athlètes. Cette information devrait être transmise sous diverses formes. Les préférences indiquées au sujet du mode de transmission de l'information sur le TC sont les suivantes : voie électronique (site Web de la CAO, courriels envoyés par les organismes de sport, site Web des bureaux de santé, etc.) et documents sur support papier (information ajoutée dans le manuel des entraîneurs, bulletins d'information, brochures, etc.).

Nos résultats peuvent servir à améliorer les connaissances des entraîneurs au sujet de la consommation de TC et des effets du TC afin qu'ils influencent les habitudes de consommation de tabac des joueurs qu'ils encadrent. Les entraîneurs devraient en apprendre davantage sur les produits du TC et sur la consommation de ces produits parce que la consommation de TC est un sujet de préoccupation croissant dans les milieux sportifs.

En comprenant mieux la perception des entraîneurs au sujet de la consommation de TC et les besoins de ces derniers en matière d'information, les professionnels de la santé publique pourront concevoir des initiatives visant à encourager les entraîneurs à jouer un rôle plus actif dans la prévention de la consommation de TC. Ces initiatives pourraient comprendre de l'information et des outils adaptés aux besoins des entraîneurs.

Comme les RRA élaborent des plans régionaux de lutte contre le tabagisme et coordonnent les activités des bureaux de santé publique faisant partie du réseau ${ }^{23}$, les résultats de cette étude peuvent leur servir à orienter leurs activités en matière de promotion de la santé et de prévention du tabagisme ainsi que celles de groupes provinciaux comme la coalition PAJ.

Les professionnels de la santé publique de l'Ontario préparent actuellement la prochaine étape de la lutte contre le tabagisme dans la communauté : la politique sur le sport et les loisirs sans tabac. Les résultats de notre étude soutiennent le mouvement du sport et des loisirs sans tabac, car ils nous renseignent sur les connaissances des entraîneurs au sujet des effets du TC sur la santé et sur la perception qu'ont les entraîneurs de la consommation de TC chez les athlètes en Ontario.

\section{Remerciements}

Les auteures aimeraient remercier la coalition Pas aux jeunes d'avoir soutenu et financé ce projet. Elles aimeraient également remercier les personnes et les organismes qui ont participé au projet :

- La Coaches Association of Ontario (CAO), en particulier Susan Kitchen et Jessica Taggio, pour leur contribution à la conception du sondage et leur expertise sur la façon de réussir à susciter l'intérêt chez les entraîneurs;

- La circonscription sanitaire du district de Haliburton, Kawartha et Pine Ridge pour son soutien dans l'analyse et la rédaction du présent rapport;

- Theresa Chambers, de la circonscription sanitaire du district de Simcoe Muskoka, pour sa contribution à la conception du sondage;

- Les entraîneurs qui ont répondu au sondage, pour avoir donné généreusement de leur temps et partagé leur expérience dans le cadre de l'étude.

\section{Références}

1. Youthography. Findings from qualitative studies into tobacco products and anti-tobacco creative development. Not to Kids Coalition; 2007. 116 p.

2. Ministère de la santé et des soins de longue durée. Normes de santé publique de l'Ontario 2008 [Internet]. Toronto (Ont.): Ministère de la Santé et des Soins de longue durée; 2008 [Consultation le 28 juillet 2010]. PDF $(493,75$ Ko) téléchargeable à partir du lien : http://www.health.gov.on.ca/french/ providersf/programf/pubhealthf/ oph_standardsf/ophsf/progstdsf/pdfs/ ophs_2008f.pdf

3. Sapundzhiev N, Werner JA. Nasal snuff: historical review and health related aspects. J Laryngol Otol. 2003;117:686-91.

4. Cooper J, Ellison JA, Walsh MM. Spit (smokeless)-tobacco use by baseball players entering the professional ranks. J Athl Train. 2003;38(2):126-32.

5. U.S. Department of Health \& Human Services (HHS). The health consequences of using smokeless tobacco: a report of the Advisory Committee to the Surgeon General [Internet]. Bethesda (MD): United States Public Health Service; avril 1986 [Consultation le 28 janvier 2011]. NIH Publication No. 86-2874. PDF téléchargeable à partir du lien : http://profiles.nlm.nih.gov/ ps/access/NNBBFD.pdf

6. Tomar SL, Winn DM. Chewing tobacco use and dental caries among U.S. men. J Am Dent Assoc. 1999;130:1601-10.

7. Colilla SA. An epidemiologic review of smokeless tobacco health effects and harm reduction potential. Regul Toxicol Pharmacol. 2010;56:197-211.

8. Enquête de surveillance de l'usage du tabac au Canada (ESUTC) 2008. Sommaire des résultats annuels de 2008. Ottawa (Ont.): Santé Canada; 2008 [Modifié le 22 janvier 2010; consultation le 28 janvier 2011]. Consultable en ligne à la page : http://hc-sc.gc.ca/hc-ps/tobac-tabac/ research-recherche/stat/_ctums-esutc_2008/ ann_summary-sommaire-fra.php 
9. Centre pour l'avancement de la santé des populations Propel. Enquête sur le tabagisme chez les jeunes 2008-2009: profil du tabagisme chez les jeunes de l'Ontario [Internet]. Waterloo (Ont.): Centre pour l'avancement de la santé des populations Propel; 2009 [Consultation le 17 août 2011]. PDF (1,45 Mo) téléchargeable à partir du lien : http://www.yss.uwaterloo.ca/ results/etj08_rapport_provincial_ON.pdf

10. Office of Applied Studies. The NSDUH report: smokeless tobacco use, initiation, and relationship to cigarette smoking: 2002 à 2007 [Internet]. Rockville (MD): Substance Abuse and Mental Health Services Administration; 19 février 2009 [Consultation le 5 mars 2009]. Consultable en ligne à la page : http://www.oas.samhsa.gov/2k9/ smokelessTobacco/smokelessTobacco.htm

11. Eaton DK, Kann L, Kinchen S, Sahnklin S, Ross J, Hawkins J, et collab. Centers for Disease Control and Prevention. Morbidity and Mortality Weekly Report: Youth Risk Behavior Surveillance-United States, 2009 [Internet]. MMWR 2010 [Consultation le 28 janvier 2011];59(SS-5):1-131. PDF (3,50 Mo) téléchargeable à partir du lien : http:// www.cdc.gov/mmwr/pdf/ss/ss5905.pdf

12. Goebel LJ, Crespo RD, Abraham RT, Masho SW, Glover ED. Correlates of youth smokeless tobacco use. Nicotine Tob Res. 2000;2:319-25.

13. Gansky SA, Ellison JA, Rudy D, Bergert N, Letendre MA, Nelson L, et collab. Cluster-randomized controlled trial of an athletic trainer-directed spit (smokeless) tobacco intervention for collegiate baseball athletes: results after 1 year. J Athl Train. 2005;40(2):76-87.

14. Castrucci BC, Gerlach KK, Kaurman NJ, Orleans CT. Tobacco use and cessation behavior among adolescents participating in organized sports. Am J Health Behav. 2004;28:63-71.

15. Melnick MJ, Miller E, Sabo DF, Farrell MP, Barnes GM. Tobacco use among high school athletes and nonathletes: results of the 1997 Youth Risk Behavior Survey. Adolescence. 2001;36:727-47.

16. Rigotti NA, Lee JE, Wechsler H. US college students' use of tobacco products: results of a national survey. JAMA. 2000;284(6):699-705.
17. DeHass DM. Substance use: NCAA study of substance use of college student athletes [Internet]. Indianapolis (IN): National Collegiate Athletic Association; 2006 [Consultation le 28 janvier 2011]. PDF (7,04 Mo) téléchargeable à partir du lien : http://www.ncaa.org/wps/wcm/connect/ $007 \mathrm{~d} 81004$ e0 dabfe9f 3 aff 1 ad 6 fc 8 b 25/ 2006_substance_use_report.pdf?MOD $=$ AJPERES $\&$ CACHEID $=007 \mathrm{~d} 81004 \mathrm{e} 0 \mathrm{dabfe}$ 9f3aff1ad6fc8b25

18. Green GA, Uryasz FD, Petr TA, Bray CD. NCAA study of substance use and abuse habits of college student-athletes. Clin J Sport Med. 2001;11:51-6.

19. Bloom GA, Durand-Bush N, Schinke RJ, Salmela JH. The importance of mentoring in the development of coaches and athletes. Int J Sport Psychol. 1998;29:267-81.

20. Parrott R, Duggan A. Using coaches as role models of sun protection for youth: Georgia's "Got Youth Covered” Project. J Appl Commun Res. 1999;27:107-19.

21. Walsh MM, Ellison J, Hilton JF, Chesney M, Ernster VL. Spit (smokeless) tobacco use by high school baseball athletes in California. Tob Control. 2000;9(2);ii32-ii39.

22. Horn KA, Maniar SD, Dino GA, Gao X, Mechstroth RL. Coaches' attitudes toward smokeless tobacco and intentions to intervene with athletes. J Sch Health. 2000;70(3):89-94.

23. Ministère de la Promotion de la santé. Document d'orientation sur la lutte globale antitabac [Internet]. Toronto (Ont.): Ministère de la Promotion de la santé (Ont.); 2010 [Consultation le 19 août 2011]. PDF (209,91 Ko) téléchargeable à partir du lien : http://www.mhp.gov.on.ca/ fr/healthy-communities/public-health/ guidance-docs/ComprehensiveTobaccoControlFR.PDF 